\title{
Delay Optimization of Center Network Cache and Performance Simulation of On-chip Network Communication
}

\author{
Huan Zhu' ${ }^{1}$, Zhiqiang Le ${ }^{2}$ \\ ${ }^{1}$ School of Physical Education, Jiangxi Institute of Fashion Technology, Nanchang, 330201, China \\ ${ }^{2}$ Basic teaching department, Jiangxi College of Foreign Studies, Nanchang, 330099, China \\ huan_zhu2013@yeah.net
}

Keywords: Cache measurement, Unstructured, IP addressing, Routing, CCN, ST.

\begin{abstract}
In order to solve the adjacent nodes repeat cache of content centric networking (CCN), this paper proposes new caching strategy based on the sojourn time (ST), and designs cooperative caching mechanism for CCN. The mechanism uses the basketball training content name as routing and uses content name to addressing, no depended on IP, with unstructured routing mode. Through the data cache simulation of single point shooting and pressing jump shooting, it can be found ST caching strategies consider the overall content and user access to content and it obtains better cache hit rate. In the two strategies of ST and ALWAYS, ST strategy gets greater delivery and reduces the delivery time, making the average delay minimum and getting the best performance.
\end{abstract}

\section{Introduction}

With the rapid development of internet technology and user increasing, many problems exist in the internet architecture of TCP/IP are also obvious, demonstrating low safety controllability, poor scalability and mobility, and service quality can't be guaranteed and other problems [1, 2]. Through the teaching process visualization display, athletes can inquiry learning to break the traditional mode of the original acceptance training, making the education teaching is from closed to open. At the same time, this makes the education training by the production talents into individualized teaching mode, and it has an important role in cultivating the students' thinking way and creative thinking, which is a subversion of the traditional education [3]. So the domestic and foreign academic circles launched a comprehensive study of the future network architecture, changing from host centered to the content as the center, and the most concern future network architecture is CCN [4,5]. After the basic idea has been proposed, many people have put forward a series of research hot spots, such as safety, content routing, congestion control and network cache, especially the research on caching mechanism in CCN network is seen as the most critical research object by the academic circles, getting extensive attentions.

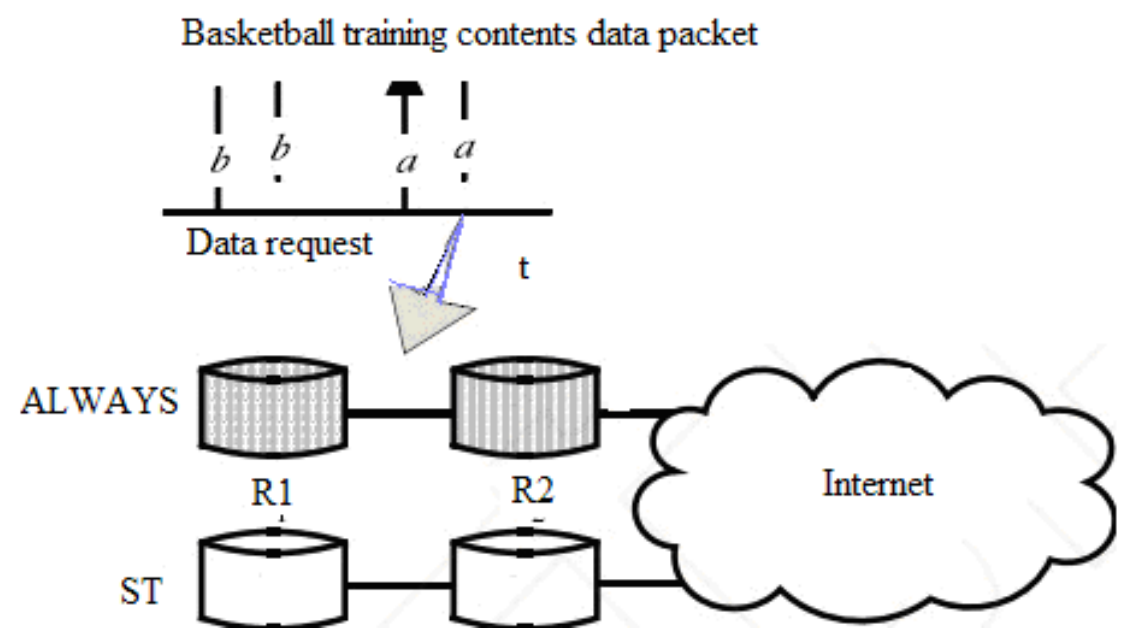

Fig.1: Schematic diagram of the basketball training project CCN cache strategy

As shown in Figure 1, the bigger of ST cache strategy buffer capacity, the greater of ST value in the cache strategy contents; the higher of the content popularity, the greater of ST value [6]. 
Obviously, by comparing the sojourn time of contents in the adjacent cache register, when the sojourn time is longer, the node can effectively reduce the number of content copies, which will increase the diversity of the AS domain.

\section{Design of CNN Caching Strategy Algorithm based on ST}

In CCN, the training content is divided into same size module, and module names are unique, which are permanently stored in one or more servers. CCN data transmission mainly uses interested packets and data packets to complete. Through the cache strategy the user sends interest packet. The routing protocol based on the name ensures that the requested routing is sent to the appropriate register, and each intermediate node will be tracking the missing node request, so the requested data is backtrack to the recipient and let the passing nodes cache data $[7,8]$. The membership function of node cache can be expressed as:

$$
P(y)=\frac{y-m}{M-m} .
$$

Among them, $M$ denotes the maximum node cache, $m$ is the minimum node cache. Node cache sequence can be written as:

$$
\left\{K_{M, m}\right\},-C_{1}<m<-C_{2} .
$$

Among them, $C 1$ and $C 2$ are cache nodes. Cache data of nodes $\left\{C_{1}{ }^{m}\right\}$ and $\left\{C_{2}{ }^{m}\right\}$ is computed according to the following formula:

$$
\begin{aligned}
& m=B-1, B-2, \ldots B-M \\
& C_{1}^{(B-1)}=\left[\frac{1}{2} C_{1}\right], C_{1}^{m}=\left[\frac{1}{2} C_{1}^{(m-1)}\right] \\
& C_{2}^{(B-1)}=\left[\frac{1}{2} C_{2}\right], C_{2}^{m}=\left[\frac{1}{2}\left(C+C_{2}^{(m-1)}\right)\right] .
\end{aligned}
$$

Through improving, the cache space is allocated.

$$
f_{o, p}{ }^{(n)}=f_{o, p}{ }^{(n-1)}-\left[\sum_{o} C_{o, p+1}{ }^{(n)} L_{p}{ }^{(n)}+\frac{1}{2}\right] .
$$

Where, $L_{p}$ is lifting coefficients, and the node cache data model is as shown below:

$$
\begin{aligned}
& L_{p 1}{ }^{i} \quad \ldots \quad L_{p}{ }^{i}{ }^{n / 2 i} \\
& \begin{array}{cccc}
L_{p 1}{ }^{2} & \cdots & & \\
L_{p 1}{ }^{2} & L_{p 2}{ }^{2} & \ldots & L_{p}{ }^{2}{ }^{n / 2 i} \\
& & \ldots \ldots . . . & L_{p n}{ }^{1}
\end{array} \\
& \Rightarrow \Rightarrow \Rightarrow \Rightarrow \Rightarrow \Rightarrow \Rightarrow \Rightarrow \Rightarrow \Rightarrow \Rightarrow \\
& \begin{array}{lllllll}
S_{1} & S_{2} & \ldots \ldots & \ldots & \ldots \ldots & \ldots . . & S_{n}
\end{array}
\end{aligned}
$$

Hypothesis that $f(x)$ is a variable function by $x$, and hypothesis that the range of $x$ is [e, $\mathrm{f}$, then the $a$ order basketball motion initialization function expression of $x$ is shown in formula (6).

$$
{ }_{e} D_{f}^{a} f(x)=f^{(a)}(x) \text {. }
$$

The model can achieve the cache strategy of ST training content center network, and the programming form is as follow:

Initialize

for $i=1$ to $M$

Update activities for all content in

if $C_{i}$ is in cache

return $C_{i}$ to the user 
Else

for $\mathrm{j}=1$ to $N$

compare $C_{i}$ and $C_{j}$;

if $C_{i}>C_{j}$

$\mathrm{xi}=C_{i}$;

end if

end for

end for

\section{Research on Basketball Training CCN Caching Strategies based on ST}

In order to effectively verify the effectiveness and reliability of the mathematical model and algorithm for CCN sojourn time cache strategy designed in second section, this paper uses the basketball training content cache to design the routing process and caching strategies, and calculates the cache hit rate and time delay characteristics [9]. The process is as shown in Figure 2.

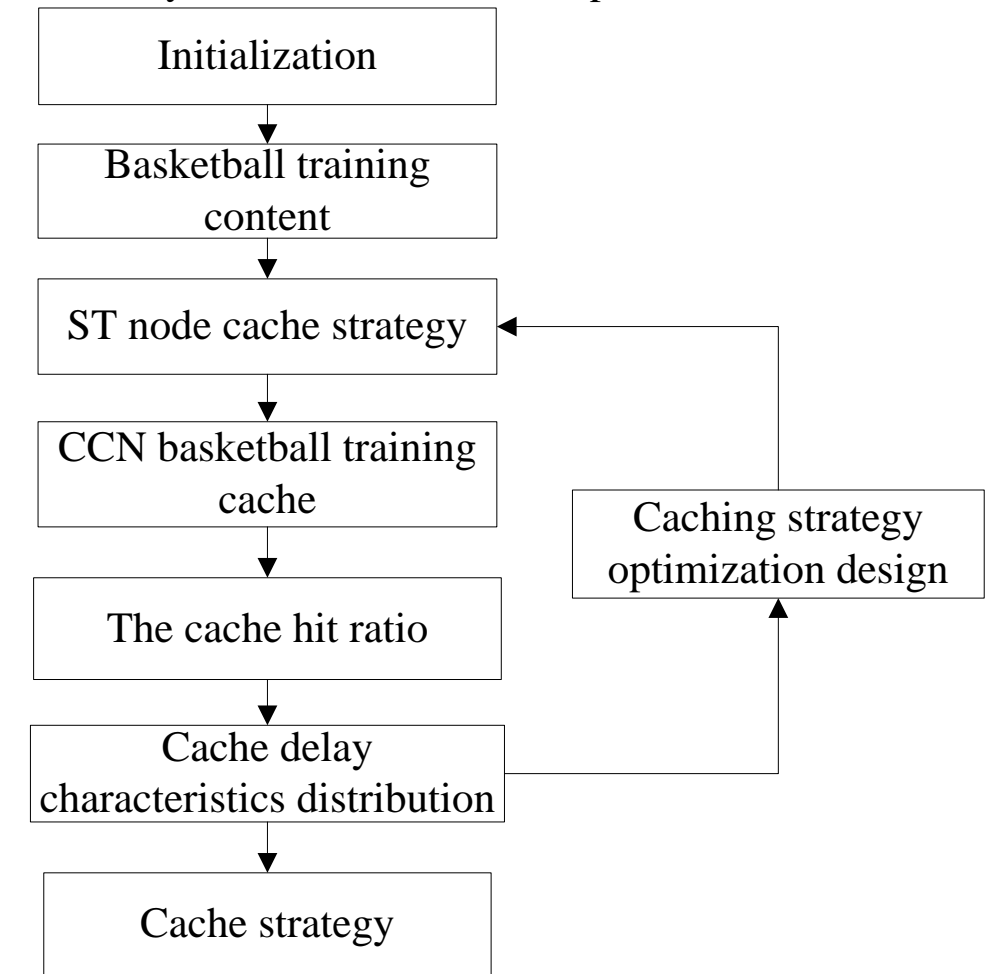

Fig.2: The center network caching strategy of training content

Figure 2 is the center network caching strategy of training content. This paper uses the basketball training content caching as an example, and optimizes the node storage design of training content through the ST node caching strategy, and the content name is as the route and it addresses through the content, so as to get rid of the dependence on the IP address to addressing [10]. The routing method uses non-structural model, and the routing process is as shown in Figure 3.

As shown in Figure 3, in CCN network, the server sends the basketball training project name to node router, the $\mathrm{E}$ router is through the network directly, and other routers establishes arriving route table according to the announcement information, and the path is $A \rightarrow B \rightarrow C \rightarrow E$ [11]. When receiving the request of the customer, router $A$ will forward the request along this path to the server to get content. 


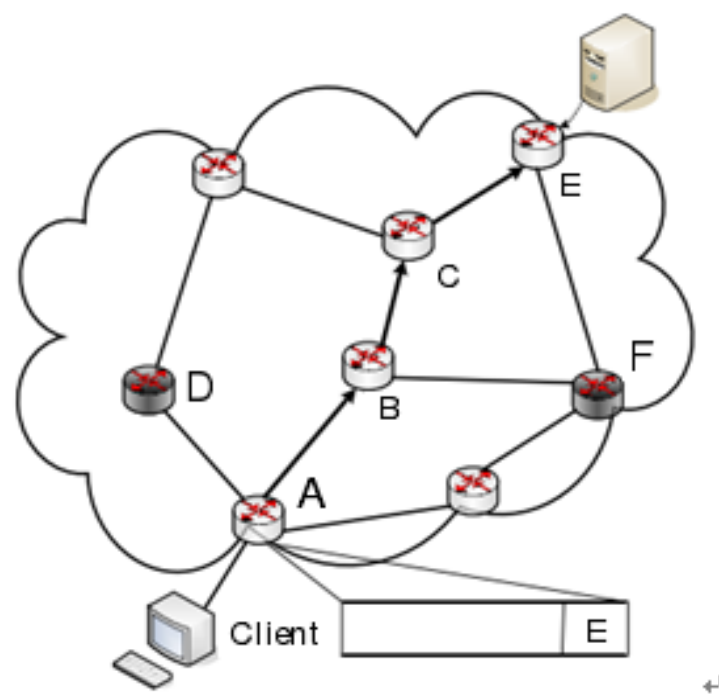

Fig.3: CCN content routing process

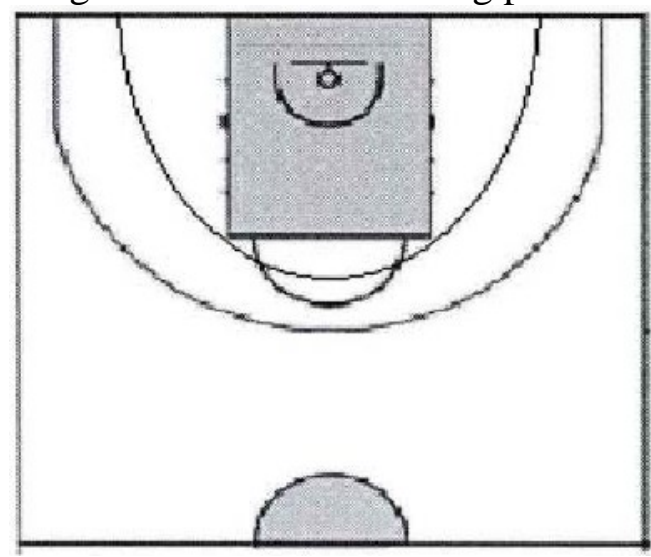

Fig.4: The hit rate testing of fixed-point shooting and pressing jump shooting

Using the test data of fixed-point shooting and pressing jump shooting as example, the tester is beyond $6 \mathrm{~m}$ of hoop projection point range, with the basketball board as a reference [12,13]. At about zero degree angles, 45 degrees angle and the front of backboard, each point they can shoot shot 5 times each, the data cache characteristics are such as shown in Figure 5.

The hit rate $/ \%$

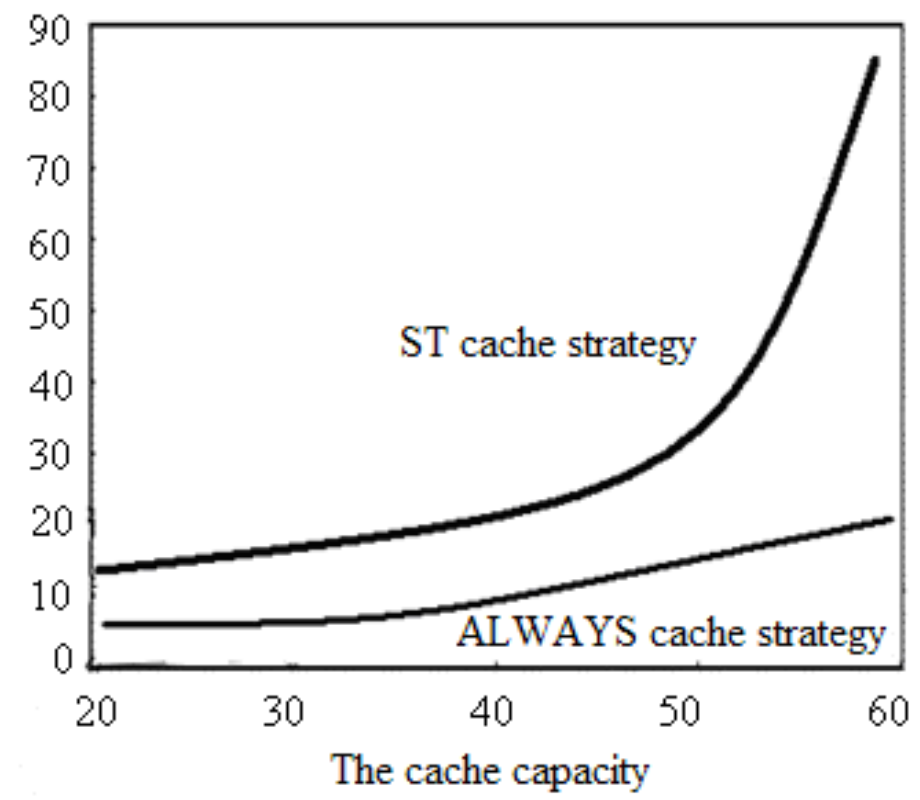

Fig.5: The curve of cache hit rate changing with the cache capacity 
Figure 5 shows the curve of cache hit rate changing with the cache capacity. The hit rate selects the average values of all the proxy server, as shown in Figure 5, the ST cache strategy considers the global content and user accessing content, and obtains better cache hit ratio, which verifies the validity of the algorithm.

The average delay time / $\mathrm{S}$

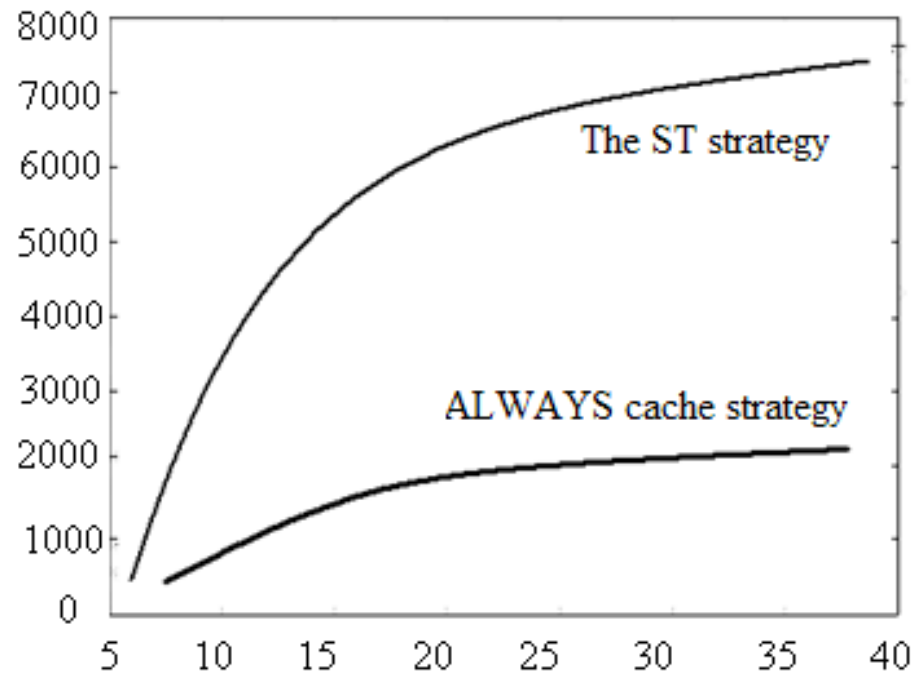

Fig.6: The average transmission delay change curve

Figure 6 shows the average transmission delay change curve. In the two strategies of ST and ALWAYS, ST strategy has minimum average delay and the best performance [14]. This is because the ST cache strategy has bigger forwarding opportunities, which reduces the time of transferring to the target and he average data delay.

\section{Summary}

With the innovation of computer hardware technology and software technology, virtual simulation technology has been applied in various fields, including sports training and basketball teaching process. This paper designs a suitable CCN to avoid repeated caching strategy of neighboring nodes, and designs the CNN cache strategy algorithm of sojourn time. This method has unstructured routing, routing, and it addressing through the content name, no dependence on IP. Using single point shot and pressing jump shot as the cache content, this paper does simulation on the hit rate and delay characteristics. Through calculation, the ST method has a better cache hit ratio, the minimum average delay and better performance. The algorithm can be extended in the future network, and reduces the storage problems brought by adjacent nodes repeat cache, which improves the performance of the system and provides a technical reference for the future network development.

\section{References}

[1] E.L. Min, Z. Chen, H.F. Xu, Y. Liang. Research progress in content centric networking. Information network security, 2012, 4(2): 56-59.

[2] L. Yang, S.W. Ma, X.X. Wang. Internet system architecture based on The content centric. Information communication technology, 2013, 4(6): 65-68.

[3] J.X. Wang, Y. Wang, Y.P. Li, J. Yuan, Z.M. Feng, Y. Ren. Analysis of the peer-to-peer network statistical characteristics based on resources popularity. Journal of physics, 2012, 2(11): 34-36.

[4] H. Tang, X. Zhou, Y.N. Han, Y.F. Tan. The evolution of next generation broadband network based on content centered. Information communication technology, 2012, 4(4): 61-65.

[5] D.Y. Wang, W.A. Zhou, Y.N. Liu. Research on content popularity focused on based on the content distribution network. Computer engineering and application, 2012, 4(6): 112-115. 
[6] Z.G. Chen, F.H. Cheng, B. Xu. Hadoop heterogeneous network policy routing based on adaptive ant colony algorithm. Journal of Wenshan University, 2013, 2(5): 87-90.

[7] C.D. Sun, M.G. Peng. Near field communication technology. ZTE technology, 2013, 2(4): 9699.

[8] W. Chen, Y. Gu, C.Y. Li, Z.X. Wu. Research review of wireless fishing access point attack and detection technology. Journal of Wuhan University (NATURAL SCIENCE EDITION), 2014, 5(1): 75-78.

[9] L.Y. Yue, Y.M. Dai. An energy optimization of WSNs non uniform routing protocol cluster. Computer engineering and application, 2012, 5(2): 52-55

[10] H.K. Zhang, H.B. Luo. The wisdom cooperative network system. Electronic journal, 2013, 2(7): 35-38.

[11] W. Su, J. Chen, H.C. Zhou, H.K. Zhang. Intelligent collaborative service mechanism in the network. Acta electronica Sinica, 2013, 2(7): 69-71.

[12] Qiu Yi. The development project practice of modern embedded systems [M]. Beijing: electronics industry press, 2011: 67-79.

[13] Zhang Jingyi, Zhao Xueyan. The design and implementation of P2P instant messaging system based on NAT [J]. Electronic design engineering, 2011, 19 (7):96-99.

[14] Liu Guozhu, Wang Honglin. The improvement congestion control algorithm of MPEG-4 realtime video transmission [J]. Microcomputer and application, 2010,18 (5): 39-44. 